Book review

\section{Gold Chemistry}

\section{Applications and future directions in the life sciences. Edited by Fabian Mohr}

Gold chemistry is difficult to do. Perhaps that is what still makes it exciting. For elements like H, P, F, Cd and Pt, all of which possess spin-1/2 nuclei, NMR spectroscopy often tells us much, for others like $\mathrm{Li}, \mathrm{Mg}, \mathrm{Al}$ and Co which possess quadrupolar nuclei, the lines are broad but NMR studies are still useful. Not so for quadrupolar ${ }^{197} \mathrm{Au}$ (the only natural isotope of gold), for which resonances are nearly always too broad and too weak to detect. So we have to resort to other techniques: Mössbauer, x-ray spectroscopy, optical methods, etc. Even theoretical methods can be revealing: the inner-shell electrons of gold move with velocities approaching the speed of light and gold shows an unusually large relativistic $6 s$-orbital stabilization (contraction) compared to its neighboring atoms in the periodic table (Chapter 4). This results in a wealth of interesting phenomena, including intermolecular $\mathrm{Au}(\mathrm{I})-\mathrm{Au}(\mathrm{I})$ (aurophilic) interactions, intermetallic compounds, unusual photochemistry, chemiluminescence, and catalysis.

The applications of gold in nanotechnology can probably be traced back over 600 years. Certainly the purple, red and pink colloids described by Andreas Cassius ("Purple of Cassius") over 300 years ago were widely used to decorate ceramics, glass and enamel, and more recently biologists have used colloidal gold nanoparticles decorated with proteins for locating, for example, antibody binding sites on cells. The surface reactivity of gold can readily be exploited e.g. by derivatization with thiols or for activating small molecules such as $\mathrm{O}_{2}$ and $\mathrm{CO}$ during catalysis (Chapter 7).

Over 100 years ago gold(I) cyanide was introduced into the clinic for the treatment of tuberculosis, subsequently to be replaced by $\mathrm{Au}(\mathrm{I})$ thiolate complexes, which then went on to be used for the treatment of rheumatoid arthritis, a use which has continued until the present. These injectable compounds were joined about 20 years ago by an orallyactive gold(I) complex auranofin, the first use of a phosphine in medicine. These uses are described by Whitehouse et al in Chapter 6. Intriguingly Au(I) cyanide is excreted by patients receiving gold therapy and apart from being present in cigarette smoke, cyanide is also a naturally-occurring ligand in the body. Some gold compounds have promising anticancer activity and one day we may well see one of them entering clinical trials.
More fundamental gold chemistry is described by Fackler et al in Chapter 1, that of gold $(I)$ with nitrogen ligands, of gold(III) with nitrogen and oxygen ligands by Cinellu in Chapter 2, of pentafluorophenyl gold complexes by Laguna et al in Chapter 3. In Chapter 5, Che and Lai provide an overview of the photophysical properties of luminescent mono- and poly-nuclear gold(I) and gold(III) complexes with potential applications in photocatalysis. Finally in Chapter 8, Coco and Espinet explain why gold(I) is a very rich source of inspiration and experimentation in liquid crystal studies.

This book is well referenced and will attract the growing number of laboratories becoming involved in gold chemistry. There is also a teaching role - good topics can be found here for undergraduate dissertations.

\section{Peter J. Sadler}

Department of Chemistry, University of Warwick, UK P.J.Sadler@warwick.ac.uk

"Gold Chemistry: Applications and Future Directions in the Life Sciences" is edited by Fabian Mohr and published by John Wiley \& Sons, March 2009. ISBN: 978-3-527-32086-8 Hardcover, 424 pages. Price $£ 130.00 / € 156.00$. www.wiley.com 